\title{
Extensions of Hadamard Codes Defined on Rings
}

\author{
Mustafa Özkan ${ }^{1, *}$, Figen Öke ${ }^{2}$ \\ ${ }^{1}$ Department of Mathematics, University of Trakya, Edirne, Turkey \\ ${ }^{2}$ Department of Mathematics, University of Trakya, Edirne, Turkey
}

\begin{abstract}
In this study, some special matrices are constructed by choosing certain elements from finite rings and certain codes are written by using these matrices.

These codes are extended to a field. Moreover these codes are classified and more good codes are written.
\end{abstract}

\section{Introduction}

Structures of cyclic, constacyclic and quasi-cyclic codes over certain finite chain rings previously were worked $(1+u)-$ Constacyclic and Cyclic codes over the ring $\mathbb{F}_{2}+u \mathbb{F}_{2}$ were practiced by Qian J., Zhang L. and Zhu S. in [4]. These subjects were extended to the ring $\mathbb{F}_{2}+u \mathbb{F}_{2}+u^{2} \mathbb{F}_{2}$ by the same authors in [5]. Defined codes in $\mathbb{Z}_{4}$ ring were obtained by Krotov D. in [1] and [2]. Hadamard Code over $\mathbb{Z}_{4}$ in particular were worked by Krotov D. in [2].

We will deal with codes defined over finite rings and binary codes.

Main aim is to obtain new and good codes. An $n$. rank square matrix $M$ that total ingredient 1 or -1 where $M M^{t}=n I$ is defined Hadamard matrix. Hadamard codes are called a code defined by Hadamard matrices. It says that using Hadamard matrices new codes could be obtained.

This study is a generalizations of results obtained in $[7,8,9]$ for the finite chain rings over $\mathbb{F}_{2}$.

\footnotetext{
*Corresponding author: mustafaozkan@trakya.edu.tr, mustafaozkan22@icloud.com
} 


\section{Basics}

Let $R_{m}$ be a ring. $C$ linear code of length $n$ in $\mathbb{F}_{2}$ is a $\mathbb{F}_{2}$ - sub space of $\mathbb{F}_{2}^{n}$. $C$ linear code of length $n$ in $R_{m}$ is a $R_{m}$ - sub module of $R_{m}^{n}$.

Let length of $C$ is $n$, it's number of elements is $M$ and it's minimum distance is $d$. Therefore $C$ be called $(n, M, d)_{-}$code.

It is known that the ring $R_{m}=\mathbb{F}_{2}+u \mathbb{F}_{2}+\ldots+u^{m} \mathbb{F}_{2}$ with $m \geq 1, m \in \mathbb{Z}$ is isomorphic to the ring

$\mathbb{F}_{2}[u] /<u^{m+1}>=\left\{x_{0}+x_{1} \cdot u+\ldots+x_{m} \cdot u^{m}+<u^{m+1}>\mid x_{i} \in \mathbb{F}_{2}, 0 \leq i \leq m, m \geq 1, m \in \mathbb{Z}\right\}$ in case $u^{m+1}=0$. It is known that the $R_{m}=\mathbb{F}_{2}+u \mathbb{F}_{2}+\ldots+u^{m} \mathbb{F}_{2}$ where $u^{m+1}=0$ is a ring

$n$. rank square matrix is called binary Hadamard matrix if it is gain from $n$. rank square Hadamard matrix $M_{n}$ placing 0 instead of 1 and writing 1 instead of -1 .

Let $n$ be an even number. Write all rows of the matrix called foregoing as a vector where have length $n$. Let us create a set putting into these vectors and respectively their ingredient to back of these vectors. Then this vectors with length $2 n$ are obtained. Write all this new vector as a codeword. If completions of codewords add to this set, the minimum distance of this code is $n$ and Hadamard code included $4 n$ elements.

The general Gray map is called as follows:

$$
\begin{aligned}
& \Phi: R_{m}^{n} \longrightarrow \mathbb{F}_{2}^{2^{m} \cdot n} \\
& a_{0}+a_{1} \cdot u+\ldots+a_{m} \cdot u^{m} \mapsto \Phi\left(a_{0}+a_{1} \cdot u+\ldots+a_{m} . u^{m}\right) \\
& \Phi\left(a_{0}+a_{1} \cdot u+\ldots+a_{m} \cdot u^{m}\right)=\left(a_{m}, a_{m}+a_{0}, a_{m}+a_{1}, \ldots, a_{m}+a_{m-1}, a_{m}+a_{0}+a_{1}, a_{m}+a_{0}+a_{2}, \ldots,\right. \\
& a_{m}+a_{0}+a_{m-1}, a_{m}+a_{1}+a_{2}, \ldots, a_{m}+a_{1}+a_{m-1}, a_{m}+a_{2}+a_{3}, \ldots, a_{m}+a_{2}+a_{m-1}, \ldots, \\
& a_{m}+a_{m-2}+a_{m-1}, a_{m}+a_{0}+a_{1}+a_{2}, \ldots, a_{m}+a_{0}+a_{1}+a_{m-1}, \ldots, \\
& a_{m}+a_{m-3}+a_{m-2}+a_{m-1}, a_{m}+a_{0}+a_{1}+a_{2}+a_{3}, \ldots, \\
& \left.a_{m}+a_{m-4}+a_{m-3}+a_{m-2}+a_{m-1}, \ldots, \ldots, a_{m}+a_{0}+a_{1}+\ldots+a_{m-1}\right)
\end{aligned}
$$

where $a_{i} \in \mathbb{F}_{2}^{n}$, for $0 \leq i \leq m$. 
The homogeneous weight $w_{\text {hom }}(r)$ of $r \in R_{m}$ is given by

$$
w_{\text {hom }}(r)=\left\{\begin{array}{l}
0 ; r=0 \\
2^{m} ; r=u^{m} \\
2^{m-1} ; \text { otherwise }
\end{array}\right.
$$

This expanded homogeneous weight function over $R_{m}^{n}$ such that $w_{\text {hom }}(r)=\sum_{i=0}^{n-1} w_{\text {hom }}\left(r_{i}\right)$ for $r=\left(r_{o}, r_{1}, \ldots, r_{n-1}\right) \in R_{m}^{n}$. The homogeneous distance $d_{\text {hom }}(a, b)$ between any different vectors $a, b \in R_{m}^{n}$ is called as $w_{\text {hom } L}(a-b)$. The minimum homogeneous distance $d_{\text {hom }}$ of $C$ is called as for each $a, b \in C, a \neq b$ $d_{\mathrm{hom}}(C)=\min \left\{d_{\mathrm{hom}}(a, b)\right\}$.

Let $C$ of length $n$ is a code in $\mathbb{F}_{2}$ and $c=\left(c_{o}, c_{1}, \ldots, c_{n-1}\right)$ be a codeword of $C$. The Hamming weight of $C$ is defined as

$$
w_{H}(c)=\sum_{i=0}^{n-1} w_{H}\left(c_{i}\right)
$$

where $w_{H}\left(c_{i}\right)=0$ if $c_{i}=0$ and $w_{H}\left(c_{i}\right)=1$ if $c_{i}=1$. The minimum Hamming distance of $C$ is defined as $d_{H}(C)=\min \left\{d_{H}\left(c, c^{\prime}\right)\right\}$ for any $c, c^{\prime} \in C, c \neq c^{\prime}$.

Therefore $C$ of length $n$ is a code in $R_{m}$ and then $\Phi(C)$ is a binary code of length $2^{m} \cdot n$

\section{Construction of Hadamard codes}

In this chapter, generator matrices were created.

Formed every elements of one row of the matrix $G^{\alpha, \beta}$ from 1 , select that the elements of the another rows from $\left\{0,1, u, \ldots, u^{m}, 1+u, \ldots, 1+u^{m}, \ldots, 1+u+\ldots+u^{m}\right\}$ set if $\beta=0$ and from $\left\{0, u^{m}\right\}$

set if $\alpha=0 . G^{\alpha, \beta}$ occured foregoing $\alpha+\beta+1$ rows. Colums of $G^{\alpha, \beta}$ be lexicographically ordered.

Examples of $G^{\alpha, \beta}$ be given below : 


$$
\begin{aligned}
& G^{0,0}=[1]_{1 x 1}, G^{0,1}=\left[\begin{array}{cc}
1 & 1 \\
0 & u^{m}
\end{array}\right]_{2 \times 2}, \\
& G^{0,3}=\left[\begin{array}{cccccccc}
1 & 1 & 1 & 1 & 1 & 1 & 1 & 1 \\
0 & 0 & 0 & 0 & u^{m} & u^{m} & u^{m} & u^{m} \\
0 & 0 & u^{m} & u^{m} & 0 & 0 & u^{m} & u^{m} \\
0 & u^{m} & 0 & u^{m} & 0 & u^{m} & 0 & u^{m}
\end{array}\right]_{4 x 8},
\end{aligned}
$$

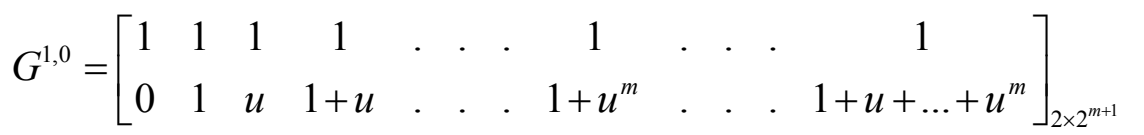

Define the code $C^{\alpha, \beta}=\left\{\left(c_{1}, c_{2}\right) \cdot G^{\alpha, \beta} \mid c_{1} \in R_{m}^{\alpha+1}, c_{2} \in \mathbb{F}_{2}^{\beta}\right\}$ which has a generator matrix $G^{\alpha, \beta}$ where $\alpha, \beta$ are integers such that $\alpha, \beta \geq 0$. The length of $C^{\alpha, \beta}$ code is $n=2^{(m+1) \cdot \alpha+\beta}$ and parameters of code $C^{\alpha, \beta}$ be $\left(n, 2^{m+1} \cdot n, 2^{m-1} \cdot n\right)$.

Theorem 3.1 : If $C^{\alpha, \beta}$ is a code generated by the matrix $G^{\alpha, \beta}$ over $R_{m}$, it's image $\Phi\left(C^{\alpha, \beta}\right)$ under the Gray map is the $\left(2^{m} . n, 2^{m+1} . n, 2^{m-1} . n\right) \ldots$ Hadamard code over the field $\mathbb{F}_{2}$.

Proof : $C^{\alpha, \beta}$ code be obtained by the matrix $G^{\alpha, \beta}$ which has dimension $(\alpha+\beta+1) \times n$ is of $C^{\alpha, \beta}=\left\{\left(c_{1}, c_{2}\right) \cdot G^{\alpha, \beta} \mid c_{1} \in R_{m}^{\alpha+1}, c_{2} \in \mathbb{F}_{2}^{\beta}\right\}$. The length of The length of $C^{\alpha, \beta}$ is $n=2^{(m+1) \cdot \alpha+\beta}$ and it has $2^{m+1} . n$ elements, i.e. $C^{\alpha, \beta}$ is a $\left(n, 2^{m+1} . n, 2^{m-1} . n\right)$ - code. Hence $\Phi\left(C^{\alpha, \beta}\right) \subseteq \mathbb{F}_{2}^{2^{m} \cdot n}$ and $\Phi\left(C^{\alpha, \beta}\right)$ is a binary Hadamard code with $\left(2^{m} . n, 2^{m+1} . n, 2^{m-1} . n\right)$ parameters.

Theorem 3.2 : The $\left(C^{\alpha, \beta}\right)^{\perp}$ is a $\left(n, \frac{\left(2^{m+1}\right)^{n}}{2^{m+1} . n}, 4\right) \_$code and it's image $\Phi\left(\left(C^{\alpha, \beta}\right)^{\perp}\right)$ under the Gray map is a $\left(2^{m} . n, \frac{\left(2^{m+1}\right)^{n}}{2^{m+1} . n}, 4\right){ }_{-}$code, in except the case $\alpha=\beta=0$.

Proof : The generator matrix $G^{\alpha, \beta}$ of $C^{\alpha, \beta}$ is the parity-check matrix of the dual code $\left(C^{\alpha, \beta}\right)^{\perp}$. The dual code of $\left(C^{\alpha, \beta}\right)^{\perp}$ contains elements $x$ of $R_{m}^{n}$ satisfied $G^{\alpha, \beta} \cdot x^{T}=0$. 
It is seen that the number of vectors be $\frac{\left(2^{m+1}\right)^{n}}{2^{m+1} \cdot n}$, minimum weight of these vectors be 4 . Hence $\left(C^{\alpha, \beta}\right)^{\perp}$ code be $\left(n, \frac{\left(2^{m+1}\right)^{n}}{2^{m+1} . n}, 4\right)$ _ code. It is viewed that $\Phi\left(\left(C^{\alpha, \beta}\right)^{\perp}\right)$ has the parameters $\left(2^{m} . n, \frac{\left(2^{m+1}\right)^{n}}{2^{m+1} \cdot n}, 4\right)$.

\section{Conclusion}

In this paper; some special matrices are constructed to obtain new Hadamard codes using the elements of the finite chain ring $\mathbb{F}_{2}+u \mathbb{F}_{2}+\ldots+u^{m} \mathbb{F}_{2}$. Certain special codes are obtained using these special matrices as generator matrices.

\section{References}

1. Krotov, D. S.,Diskretn. Anal. Issled. Oper. Ser.1., 7, 4. 78-90,(2000)

2. Krotov,D. S. ,Procs. of the International Workshop on Coding and Cryptography, Paris, 329-334,(2001)

3. Vermani, L. R., Chapman Hall, India., (1996)

4. J.F.Qian, L.N.Zhang, S.X. Zhu, Applied Mathematics Letters, 19,820-823,(2006)

5. J.F.Qian, L.N.Zhang, S. X. Zhu,IEICE Trans.Fundamentals, E89-a, no 6,18631865,(2006)

6. W. C. Huffman and Vera Pless, Fundamentals of Error Correcting Codes, Cambridge, (2003)

7. M. Özkan and F.Öke, App Mathematics and Inf. Sci. 10 (2), 701-704.,(2016)

8. M. Ozkan, F. Oke, General Letters in Mathematics, 2(1), 110-118, (2017)

9. M. Ozkan, F. Oke, AIP Conf. Proc.,1926, 020035-1-020035-3,(2018). 